

\title{
In-flight experiment for combined planar antennas and solar cells (SOLANT)
}

\author{
$\begin{array}{lll}\text { S. Vaccaro } & \text { C. Pereira } & \text { J.R. Mosig } \\ & \text { P. de Maagt }\end{array}$ \\ ${ }^{1}$ JAST Antenna Systems SA, PSE-EPFL, CH-1015 Lausanne, Switzerland \\ ${ }^{2} R U A G$ Aerospace, Wallisellen 8304, Switzerland \\ ${ }^{3}$ Laboratory of Electromagnetics and Acoustics (LEMA), Ecole Polytechnique Fédérale de Lausanne (EPFL), CH-1015 Lausanne, \\ Switzerland \\ ${ }^{4}$ Electromagnetics Division, European Space Agency, 2200 AG Noordwijk, The Netherlands \\ E-mail: stefano.vaccaro@jast.ch
}

\begin{abstract}
An in-flight experiment to validate advanced solar antenna structures (SOLANT) in space is presented. Two different antennas have been designed and manufactured, one for global positioning system (GPS) reception and one for S-band beacon transmission. Both units use slot aperture radiators embedded in identical mechanical structures. The GPS antenna has been designed to generate an omni-directional radiation pattern with righthanded circular polarisation thus fulfilling NavStar specifications. The S-band beacon antenna is based on a four-element circularly polarised array providing $6 \mathrm{dBic}$ of gain. These antennas are integrated within two solar panels, which form the upper layer of the antenna structure. The two panels are identical, make use of gallium arsenide (GaAs) solar cells arranged in two strings and provide a peak power of $10 \mathrm{~W}$ each. The SOLANTs were launched, fixed to a Cosmos payload adapter and remained operative for 2 years, well beyond their expected lifetime. This study summarises the design and performance tests performed prior the launch and during the orbital operation.
\end{abstract}

\section{Introduction}

Since its first proof-of-concept introduction in 2000 [1], the SOLar ANTenna (SOLANT) has evolved towards advanced designs, which have been further refined [2-4], thus leading to a sophisticated space-qualified flight model, successfully flown as described in this paper. The underlying idea in SOLANT is the combination of planar antennas and solar cells in a single structure and therefore an increase in the surface available on a spacecraft for other instruments or components. An added benefit is the simplification of the satellite structure by combining two main functions, telecommunication and power generation, in one compact component. Most satellite systems use separate solar cells and antennas, which are often in competition for the available surface in satellite systems. This was maybe not an unsolvable problem for traditional satellites, but it is becoming a serious trade-off issue in commercial mini-, micro- and nano-satellites, where size and weight should be as small as possible. It is also an issue for operational and next-generation satellite telecommunication systems that often require multiple beam capability. The conventional way to achieve this is to use multiple apertures (typically four reflectors) and a single feed per beam design. The power demands for these systems lead to relatively large solar arrays. Being able to combine both solar and antenna arrays would safeguard advantages especially in terms of mass and spacecraft accommodation.

More specifically, the SOLANT concept, deeply combining planar antennas and solar cells, could be particularly advantageous in the following types of space missions:

- Missions where there is a natural coherence between sun direction and antenna boresight. This option may be very attractive for the European Space Agency (ESA) Future Science Program both in terms of deep space missions to the outer planets (Mars and beyond) and missions at the L2 Lagrangian point of the earth-sun system. At this 
location, the sun, the earth and the moon are all located behind the payload which points away from the sun.

- Missions where there is an advantage to conform the antenna to the spacecraft body (e.g. spinning satellites). An obvious example of such a mission is a one-axis stabilised satellite. In this case, a large cylindrical part of the satellite surface can be covered by integrated solar cells and radiating elements. Each of them faces halftime the sun and the earth. The subarrays of the integrated antennas with solar cells should not be fed all at the same time, but an electronic feeding network will have to allow for a continuous rotation of the beam that opposes to the satellite spinning.

- Missions requiring very large antennas and/or solar arrays.

- Missions requiring very small spacecrafts.

For all of the above missions there is the option to save 'real estate' by combining two functions maintaining performance equal or to increase capability (e.g. to offer communication in a non-nominal situations). In several cases, SOLANT could even be a mission enabling solution.

\section{Payload description}

In the past, SOLANT structures have been tested on earth and several configurations have been explored. These include patch and slot radiators with both gallium arsenide (GaAs) and amorphous silicon $(\mathrm{a}-\mathrm{Si})$ solar cells $[3,4]$. The feasibility of using the solar cell itself as a radiating patch, achieving ultimate integration, has also been demonstrated $[5,6]$. These studies have brought the technology to a maturity stage where an in-flight test of the concept was the next logical step. The validation of this technology on a Cosmos payload adapter [7] has been performed within the framework of the ESA project 'Advanced SOLar ANTennas' (A-SOLANT).

The objective of the mission was to perform a complete test of the SOLANT capabilities and an evaluation of the use of different antenna configurations in the space environment. Two antenna types have been manufactured and integrated using a solar panel as uppermost layer (see Fig. 1). This panel used GaAs cells for power generation. The first one antenna has been designed for GPS reception whereas the second one acts as an S-band beacon. This allows transmission and reception capabilities to be tested. The main challenge in the A-SOLANT project has been the development of a design suitable for both units and the verification of solar panel and antenna functionality in a single structure.

The Cosmos payload adapter [7] is depicted in Fig. 2. This solution was essentially selected for economical reasons, since this adapter is placed on the upper stage of the rocket used to launch other satellites. After the detachment of all the

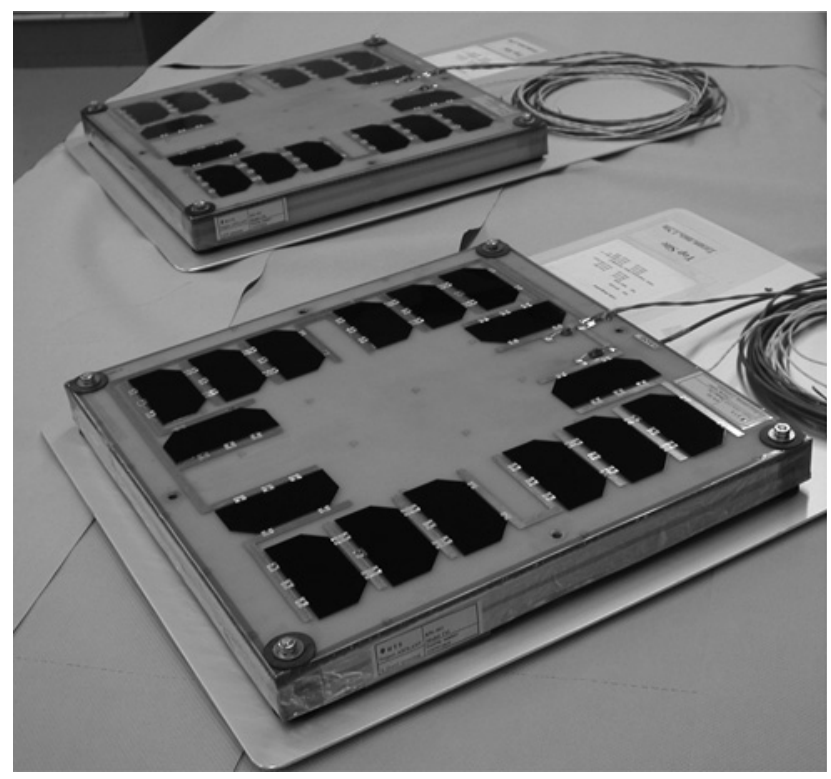

Figure 1 Finished space-qualified SOLANT units

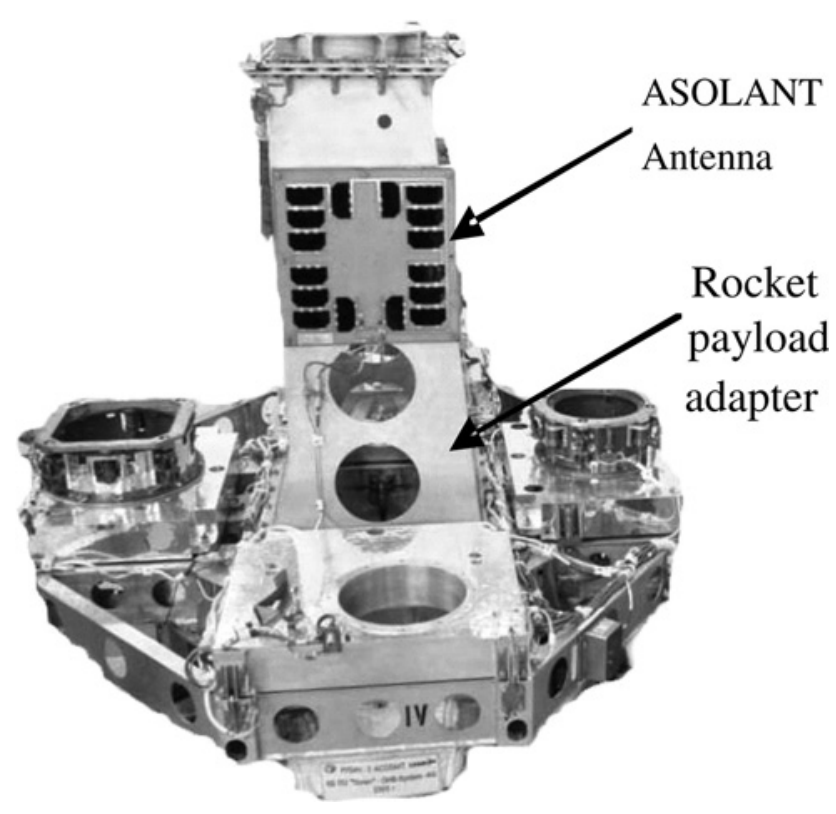

Figure 2 Cosmos payload adapter showing location of the antenna

satellites, the payload adapter remains in a low earth orbit and can be used to perform in-flight experiments. In our case, the SOLANT experiment was bolted to this platform adapter and designed to remain in orbit with it. The experiment was intended to send signals to a ground station and to allow periodical monitoring of GPS reception and power generation. SOLANT operated successfully during 2 years in space, thus largely surpassing its original life expectancy of a few months.

The complete payload consisted of two solar antennas, a GPS receiver and an S-band transmitter. A front view can be seen in Fig. 2 (the second antenna is on the opposite 
side of the payload adapter). The telecommunication system of the payload included as well all peripheral equipment to connect the antennas and in particular the following instruments:

1. GPS receiver: The GPS receiver allows the estimation quality of reception of the respective A-SOLANT antenna. The GPS data are transmitted via the Orbcomm system using the S-band beacon.

2. Orbcomm modem: The Orbcomm modem is used as satellite remote control and as backup system for the data downlink. The modem is connected to a dedicated antenna.

3. S-band beacon transmitter: The S-band beacon is used for the evaluation of the solar antenna performances in transmission operations. The beacon will be used to transmit data such as position (GPS), orientation (measured using a magnetometer), solar cell generated power and measured temperature.

With this experimental set-up, the mission was designed not only to test the SOLANT technology but also to ascertain the benefits provided to the overall payload by the use of these integrated solar antennas.

\section{Antennas' mechanical structure and tests}

The mechanical structure of the two solar antennas can be seen as a double-tier-layered sandwich structure (Fig. 3). The upper side of this structure is a glass fibre reinforced epoxy plate that supports the solar cells. This plate is separated by a $5 \mathrm{~mm}$ honeycomb core from the actual antenna. The main antenna layer is a RT Duroid 5870 substrate, where the microstrip lines and the slot radiators are etched. In its lower tier, the antenna substrate layer is joined to the $0.5 \mathrm{~mm}$ thick aluminium base plate by a $30 \mathrm{~mm}$ thick polyurethane open cell foam core. The antenna integrates attachment points, power and data cables as well as temperature sensors and protection diodes. Fig. 4 depicts the cross-section of the SOLANT structure illustrating the different layers.

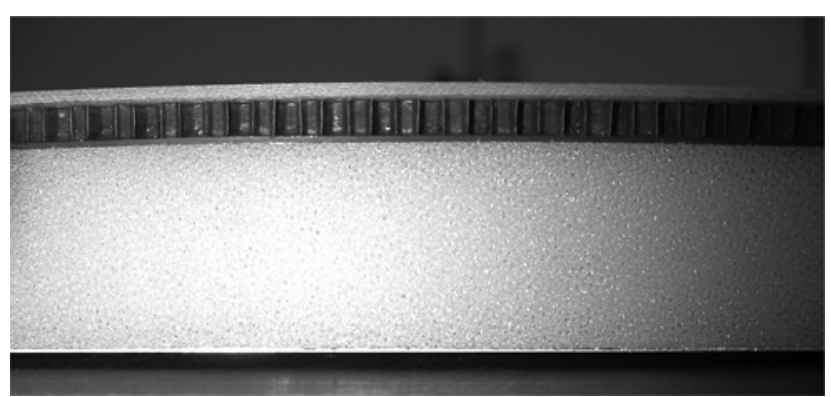

Figure 3 Generic cross-section of a solar antenna showing its mechanical structure. The picture also evidences the antenna curvature generated by the glue curing

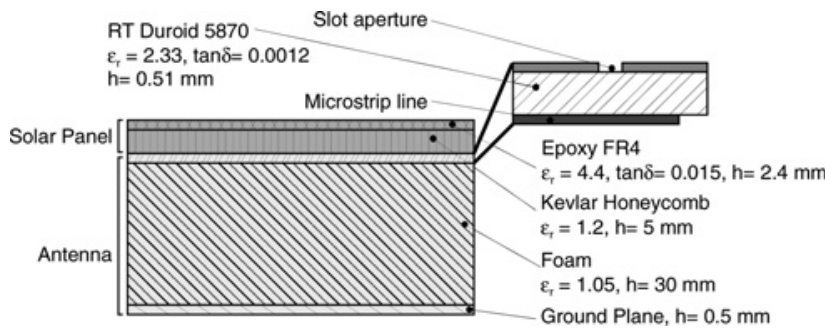

Figure 4 Cross-section of a typical SOLANT structure showing both the antenna and solar parts

The whole SOLANT structure went through vibration, electrical, antenna performance, solar tests and heat soaking. The vibration test simulated the qualification launch spectra from the Cosmos launcher matching it in duration whereas the electrical, performance and solar tests benchmarked performance of the equipment before and after vibration. Structural integrity was verified by low-level sine sweeps.

In addition, a depressurisation test was performed on an identical structure in order to verify integrity of foam and honeycomb because of exposure to high vacuum environments.

The foam separating the antenna from the support structure had to meet several criteria: low dielectric strength, open pore structure and mechanical damping. The selected foam was an open cell foam based on a polyurethane matrix with a high compressive strength to avoid cell collapse in vacuum. The foam had a high damping coefficient (measured at 6\%). This greatly reduced the peak loads on the solar cells.

Solar tests were done to measure power delivered by the cells; electrical tests to verify isolation, grounding and electrical continuity of the electrical connection; finally, heat soaking tests were performed to verify compliance of the hardware to the expected thermal environment.

All tests were successfully carried out; integrity of SOLANT and compliance to specifications were verified during the tests campaign.

The illumination tests have been performed at SUPSI in Lugano, Switzerland.

\section{Solar panels}

The structures of the solar panels used for the two antennas are identical. The panels are based on a glass fibre reinforced epoxy plate on which the solar cells interconnecting lines are etched. The GaAs triple junction solar cells are directly glued on the plate and connected to the lines via bridging elements.

The cells form two strings each of which supplies $9 \mathrm{~V}$ at $500 \mathrm{~mA}$ for a total of $9 \mathrm{~W}$ per panel. Protection diodes are 
Table 1 Measured maximum power of the solar panels

\begin{tabular}{|l|c|c|}
\hline \multirow{2}{*}{} & \multicolumn{2}{|c|}{ Power measurements, W } \\
\cline { 2 - 3 } & $\begin{array}{c}\text { GPS } \\
\text { antenna }\end{array}$ & $\begin{array}{c}\text { S-band } \\
\text { antenna }\end{array}$ \\
\hline solar panel alone & 11.0 & 10.8 \\
\hline antennas fully assembled & 10.9 & 10.8 \\
\hline $\begin{array}{l}\text { antennas after vibration } \\
\text { test }\end{array}$ & 11.0 & 10.2 \\
\hline
\end{tabular}

fixed at the end of each string. The solar cell performance was measured during the different preparation stages by flash tests. The power delivered by each panel is summarised in Table 1.

A square zone of $170 \mathrm{~mm} \times 170 \mathrm{~mm}$ has been left free of solar cells at the centre of the panel (Fig. 5). The thin slot antennas are located directly below this square. Other configurations are possible if an increase in the area covered by solar cells is desired. In previous tests [4], it has been demonstrated that solar cells can be placed very close to the slot antenna's edges without adversely affecting their electromagnetic performance.

\section{GPS antenna}

The GPS antenna has been based on slot aperture radiators in order to offer the maximum compatibility with the solar panels [3]. The GPS antenna has been designed to generate a broad beam with low directivity. It is circularly polarised and operating in the GPS L1 frequency of $1.575 \mathrm{GHz}$.

The layout of the GPS antenna is shown in Fig. 6. The antenna consists of four linearly polarised slots, each excited through a single microstrip line. Each slot is turned and

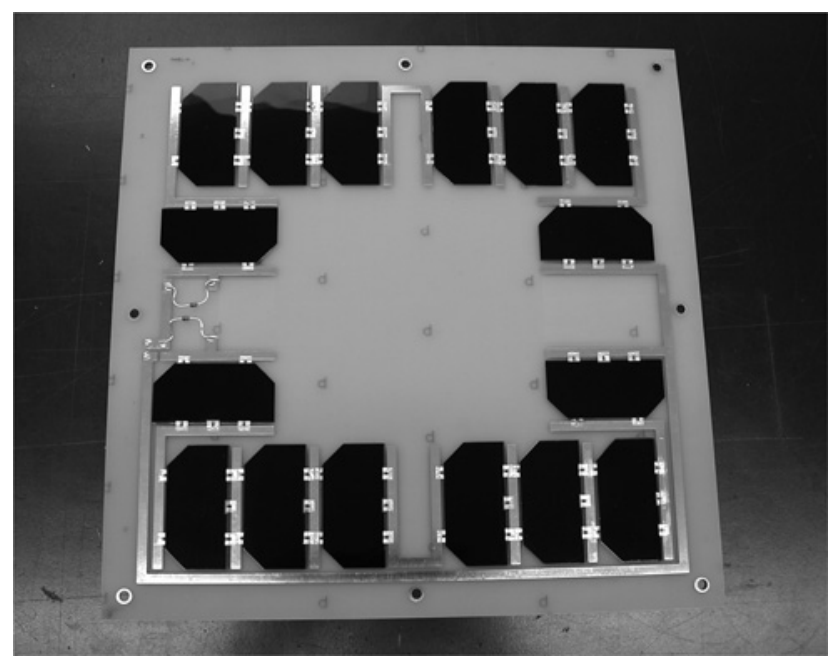

Figure 5 Top view of a solar panel forming the SOLANT upper layer, showing the DC connections

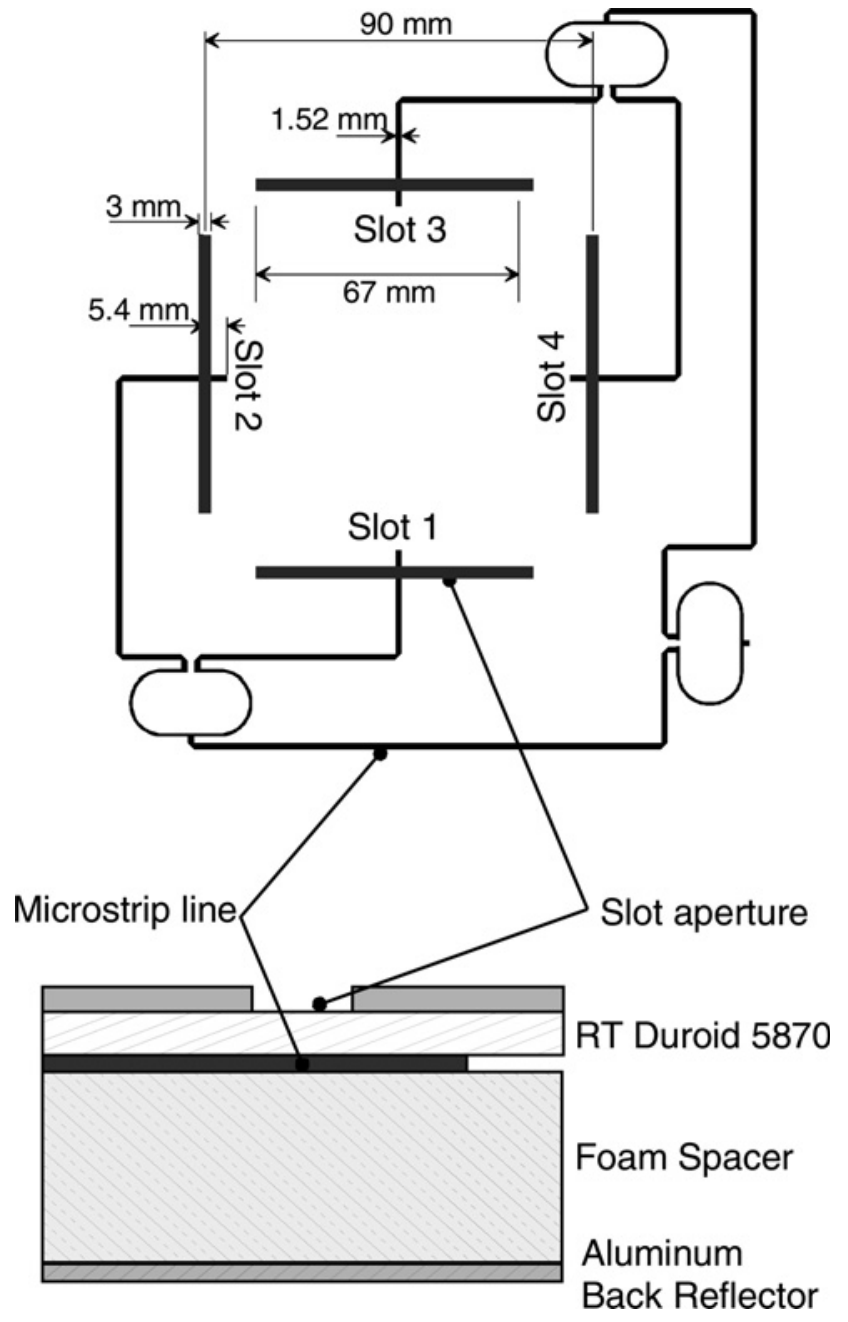

Figure 6 Antenna part: layout and cross-section

phase shifted by $90^{\circ}$ with respect to its neighbour. The feeding network of the slots has been designed using Wilkinson power dividers and delay lines. By adjusting the length of the different feeding lines, each element is fed with the correct phase slot $1: 0^{\circ}$; slot 2 : $90^{\circ}$; slot $3: 180^{\circ}$; and slot 4: $270^{\circ}$.

Below the feeding network, a back reflector has been placed at $30 \mathrm{~mm}$ from the feeding lines. This distance was imposed by mechanical constraints of the antenna structure. The distance between the slots and the back reflector is considerably below the optimum of $\lambda / 4$ $(\sim 50 \mathrm{~mm}$ at $1.575 \mathrm{GHz})$ resulting in lower antenna efficiency. This loss in efficiency has been estimated by simulation to be $25 \%$. Nevertheless, the maximum gain was predicted to be higher than $5 \mathrm{dBic}$, largely within the requirements of the GPS system.

\subsection{Measurements of the GPS antenna}

The GPS antenna flight model has been measured during all phases of its integration with the solar panels. The first measurement series has been performed by using copper patches simulating the solar cells (measurement with 


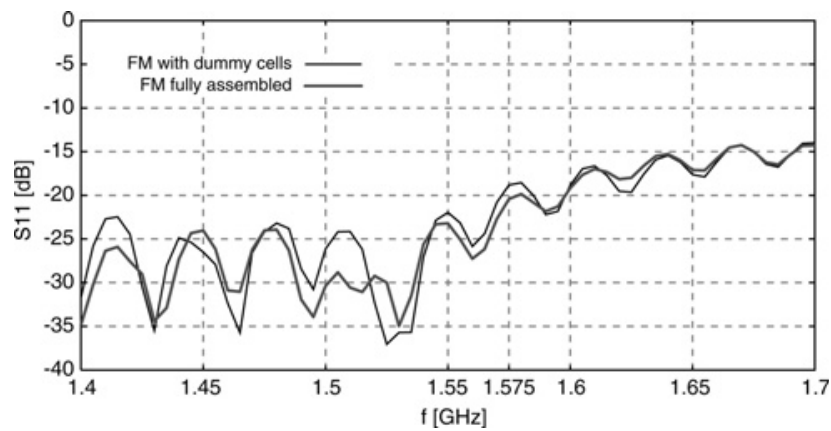

Figure 7 S11 measurements of the GPS antenna

dummy solar cells). This first measurement allowed testing the antenna before integration with the solar panel. The second measurement configuration consisted in the antenna with the solar panel in place but not yet glued. Once the GPS antenna flight model was assembled, it has been remeasured in order to confirm its functionality.

Two of these measurements of the S11 values of the GPS antenna can be seen in Fig. 7. The difference between the measurements with 'dummy cells' and with the final panel is within measurement accuracy.

The radiation characteristics of the GPS antenna flight model were also measured in the different configurations described previously. The antenna radiation patterns were measured using the spinning dipole technique [8] in order to evaluate the circular polarisation characteristics of the antenna.

Two scans were performed in the horizontal and vertical planes. These are shown in Fig. 8 for the case of measurements with dummy cells and in Fig. 9 for the assembled unit.

A slight difference appears in the radiation pattern symmetry between the first and the final measurements. The difference is essentially because of the presence in the complete assembly of all the solar cells, their connectors and wires. An accompanying gain reduction was observed between the two measurements but this offset is within the margin of the link budget for this antenna.

The degradation is mainly caused by the physical link (cable and connectors) between the antenna and the receiver. Although it would have been desirable to improve the system performance, the tight launch window prevented any further optimisation.

\section{S-band antenna}

The S-band antenna has been designed to ensure a beacon communication link between the payload and the ground station. The calculated link budget has shown that a near omni-directional antenna (minimum transmission gain required is $0.2 \mathrm{dBic}$ ) would ensure the reception of the signal by the ground station. In order to allow sufficient
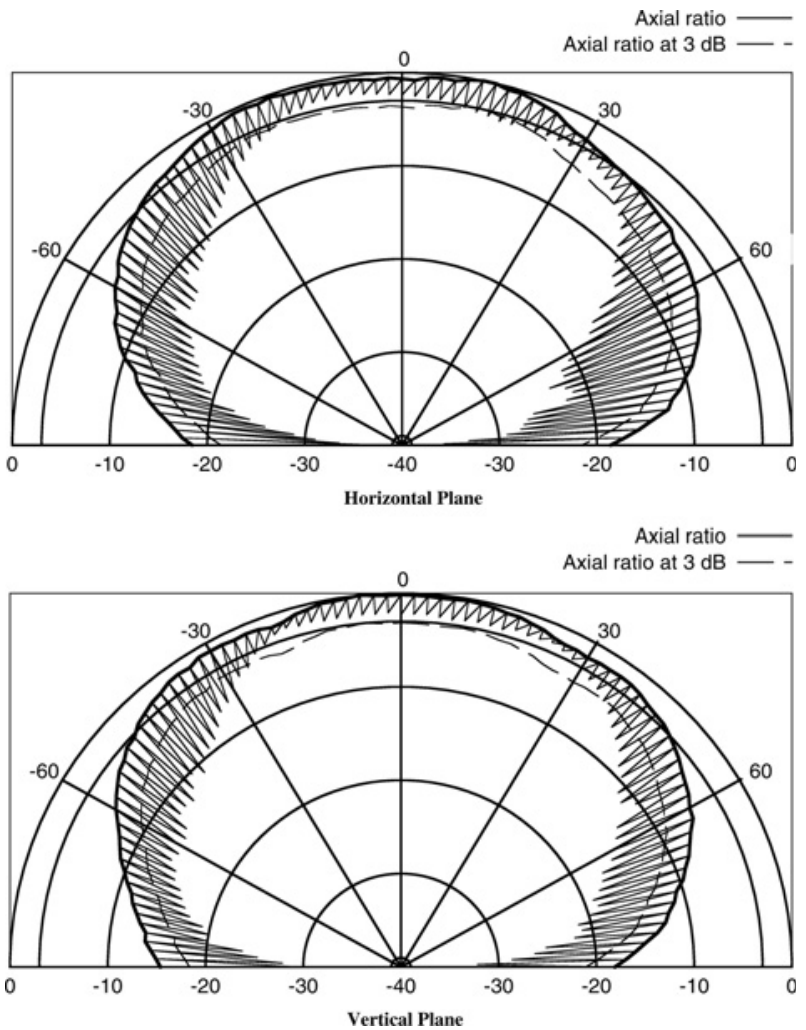

Figure 8 Measured radiation pattern of the GPS antenna on the flight model with dummy solar cells

Max gain at $1.575 \mathrm{GHz}$ is $5.5 \mathrm{dBic}$

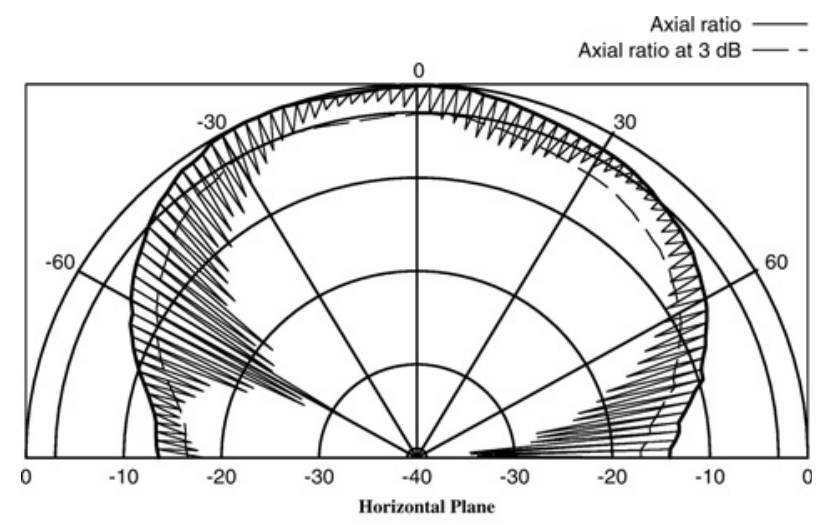

Axial ratio Axial ratio at $3 \mathrm{~dB}$

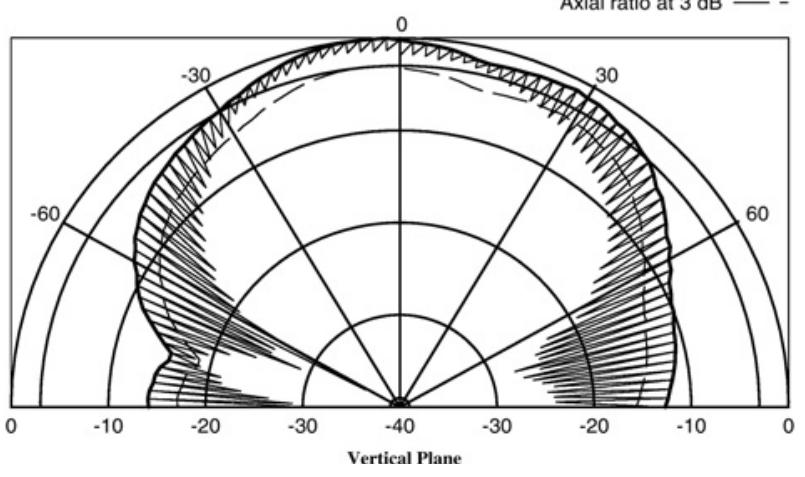

Figure 9 Measured radiation pattern of the GPS antenna on the fully assembled flight model

Max gain at $1.575 \mathrm{GHz}$ is $3 \mathrm{dBic}$ 
margin in this link the S-band transmission antenna has been designed to have a gain greater than $5 \mathrm{dBic}$.

The main specifications of the S-band antenna are

Frequency band

Polarisation

Impedance

VSWR

Gain

\section{$2.4-2.45 \mathrm{GHz}$ circular RHCP \\ $50 \Omega$ \\ less than $1.5: 1$}

greater than $5 \mathrm{dBic}$

For the S-band antenna, an array of four basic elements has been selected. Each element is composed by two orthogonal slots fed by two microstrip lines. The two slots are phase shifted by $90^{\circ}$ in order to generate circular polarisation.

The complete array is composed by four double-slot elements placed in a $2 \times 2$ configuration and sequentially rotated [9], as depicted in Fig. 10. Each element is orthogonal to the other slot element and is fed with a phase shift of $90^{\circ}$. Circular polarisation is further improved by ensuring the minimum of mutual coupling in designing the slot position.

This structure has been selected because it ensures a higher directionality with respect to the configuration used for the GPS antenna.

The measurements of the S-band antenna have been carried out in parallel with the measurements of the GPS antenna. Therefore the measurements have been performed in the same three configurations described previously namely without solar cells, with solar cells placed on top but not glued and in final configuration.

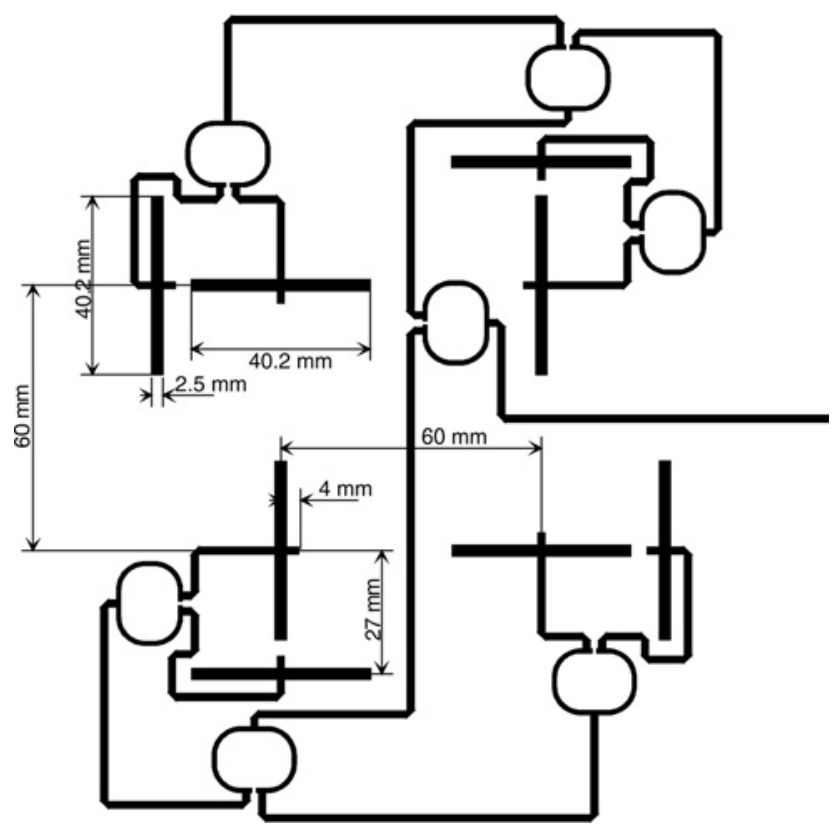

Figure 10 Layout of the feeding network and the slots' arrangement for the S-band antenna

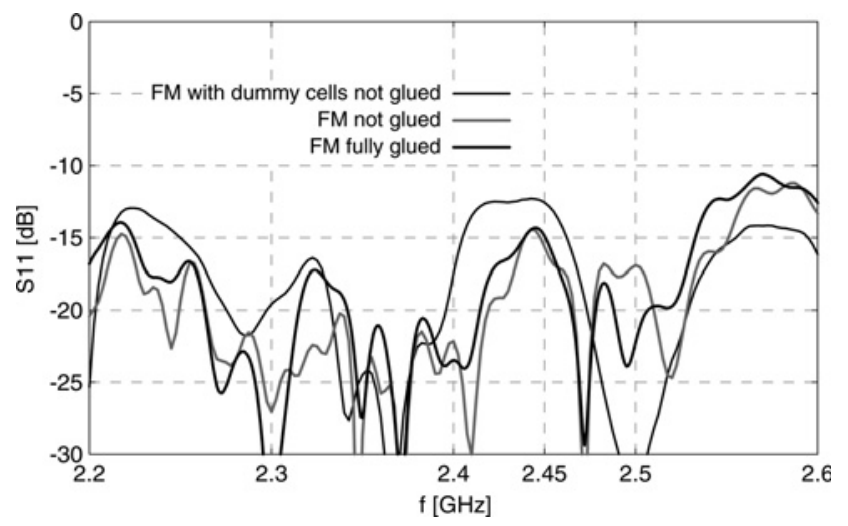

Figure 11 S11 measurements of the S-band antenna

Fig. 11 shows the results of the S11 measurement of the S-band antenna and the difference is again within measurement accuracy. From these measurements, it appears that the S11 without solar cells shows good performance meeting the required bandwidth. This performance is also achieved in the flight configuration.

The radiation characteristics of the S-band antenna have been measured in the different configurations and are shown in Figs. 12-14.

The measurements on the radiation pattern show a minor degradation of the circular polarisation quality in the case
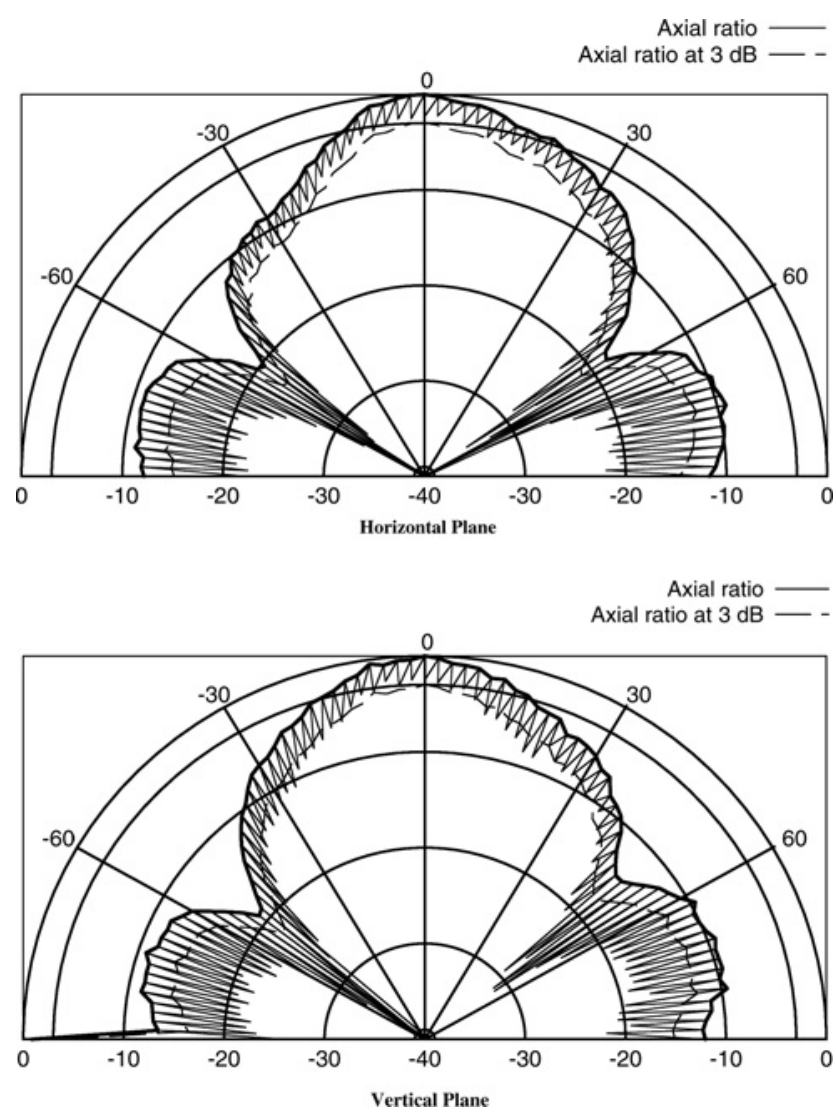

Figure 12 Measured radiation pattern of the S-band antenna on the flight model with dummy cells 


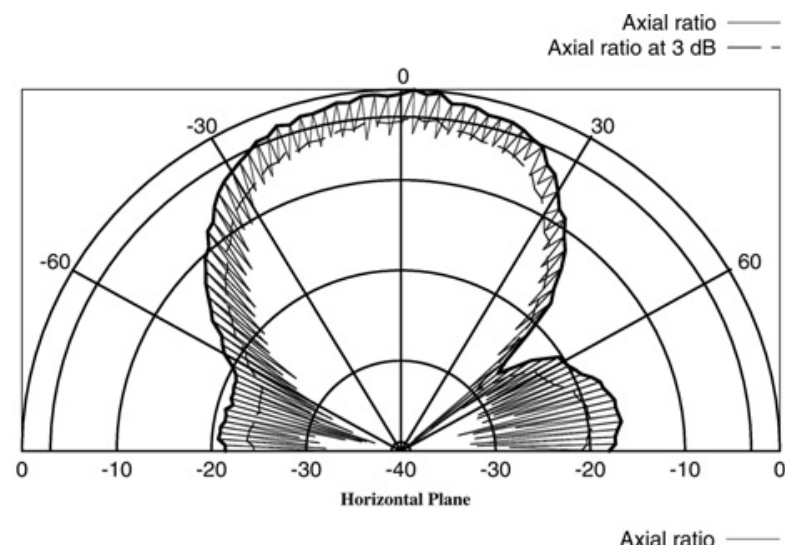

Axial ratio at $3 \mathrm{~dB}$

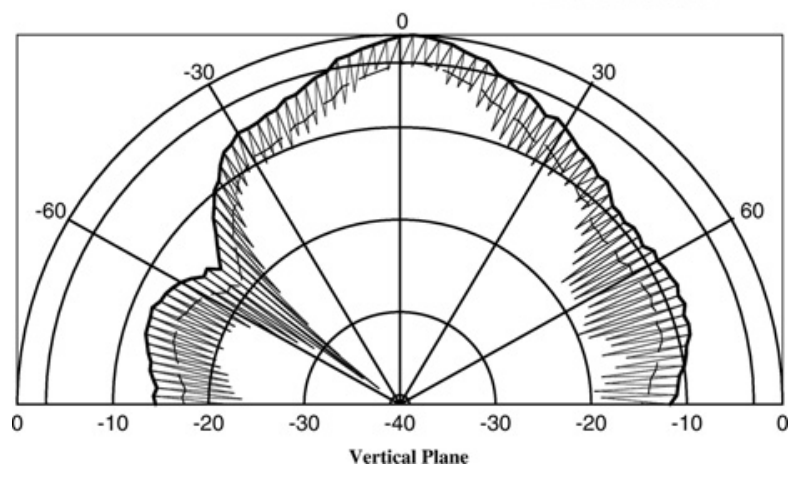

Figure 13 Measured radiation pattern of the S-band antenna on the flight model with final solar panels not yet glued
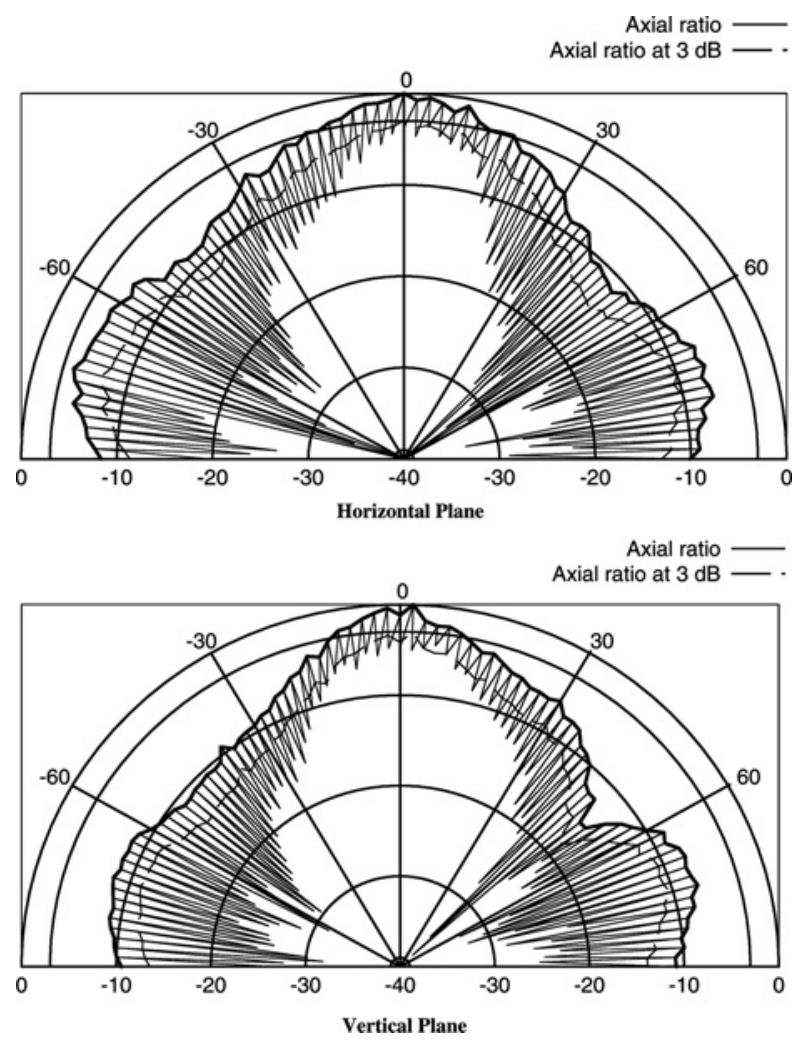

Figure 14 Measured radiation pattern of the S-band antenna

Flight model with solar panels glued

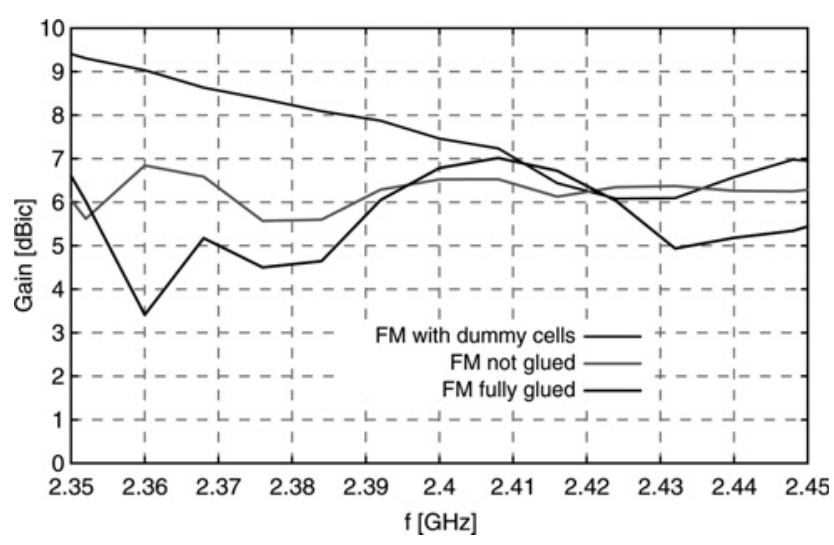

Figure 15 Measured gain of the S-band antenna flight model

when the solar panel is fully glued (Fig. 14) solar panel. This effect has neither been observed in the measurements carried out with the dummy solar cells nor in the measurements on the flight model of the solar panel flight model when the cells were not glued. So, the degradation cannot be attributed to the interaction between the antenna and the solar cells, but to a curvature of the antenna caused by a thermal expansion mismatch between the solar array carrier and the lower fixation plate during the curing of the adhesive layer at $140^{\circ} \mathrm{C}$.

This effect can be appreciated in Fig. $3 b$ and could be minimised by preheating of the components. In any case, the observed degradation has negligible impact on the link budget.

The measurements of the antenna gain, depicted in Fig. 15, show the variation of values during the different manufacturing stages. The antenna provides a gain larger than $5 \mathrm{dBic}$ over the complete transmission frequency band.

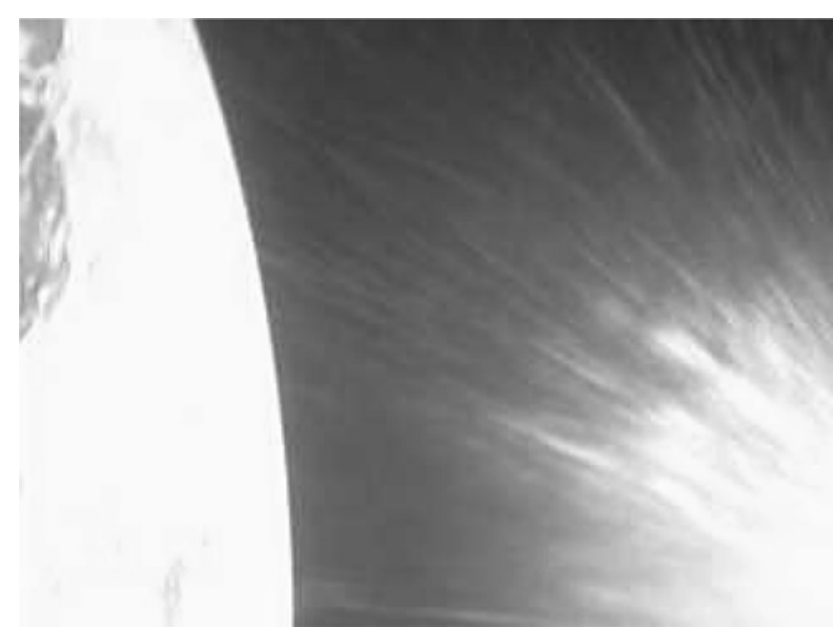

Figure 16 View of the earth

Picture taken by the A-SOLANT experiment camera 

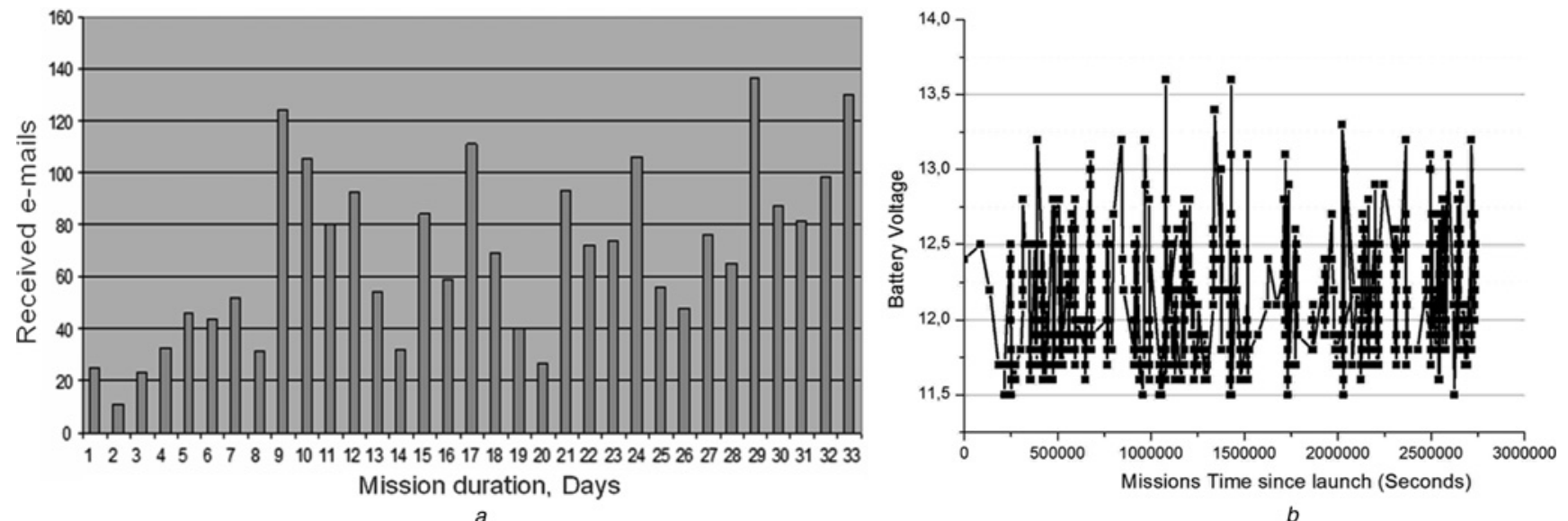

$b$

Figure 17 Example of satellite housekeeping data received at the ground station

a Number of received e-mails

$b$ Battery voltage status during the first month of experiment

\section{Flight experiment results}

The SOLANT experiment was launched on 27 October 2005 from Plesetzsk in Russia and during 2 years SOLANT has operated properly by sending useful experiment data. Data messages were received through Orbcomm satellites and their associated gateways located in, among other countries, the USA, Italy, Kazakhstan, Korea, Japan and Brazil. SOLANT is charging the batteries whenever the launch vehicle upper stage is not in eclipse and the battery condition is regularly reported to the ground. Large numbers of magnetometer measurements are being received, enabling ground control to calculate the orientation of the launch vehicle upper stage. Housekeeping data, such as temperature, voltage and attitude, have been received daily. Pictures taken with a low-resolution camera have also been successfully sent (see Fig. 16).

The data downloaded also show that the solar panels operate properly and are supplying power to the complete experiment. During the experiment, no degradation of the performances of the solar panels has been observed and the power budget of the payload remains constant (Fig. 17).

The GPS antenna and the S-band antenna have shown their functionalities even under extremely unfavourable conditions because of the failure in the detachment of one of the satellites carried by the payload adapter. One of the satellites remained attached to the adapter in front of the GPS antenna blocking its view of the sky. This event had an important impact on the GPS antenna functionality and the power budget of the mission that has been reduced by half because of the unexpected shadow.

Even so, the GPS antenna is able to receive signals from the GPS network. Signals from the S-band transmitter have also been received by amateur radio ground stations in Germany, even if the transmitter can only be switched on for very short periods because of the power limitations.

\section{Conclusions}

The in-flight experiment described in this paper has validated the feasibility of combining antennas and solar cell arrays in a single unit for space applications. The module delivered a peak power of $10 \mathrm{~W}$ with a GPS antenna fully compliant with NavStar specifications. The S-band antenna safely operated within the link budget.

The performance of the two antennas has exceeded all expectations; they operated well beyond their expected lifetime in the uncontrolled environment of a free floating platform in space. The large amount of data collected from the long duration flight experiment has allowed detailed monitoring and unquestionable verification of the SOLANT performance in space. This validation on a simplified platform opens the door to other applications of SOLANT in space exploration. Several scenarios contemplating its use are currently in the conceptual stages or being baselined for future missions (e.g. providing TT\&C on the rover for the EXOMARS mission).

As frequently happens with technological developments fostered by the needs of space exploration the SOLANT concept can also be applied to earth-bound applications. For these, where pricing is a major driving factor, less efficient but much cheaper flexible solar cells could be used without degrading the antenna performance.

\section{Acknowledgments}

The work reported here was carried under contract no. 12879/98/NL/NB CCN 1 of the ESA. The authors wish to acknowledge the support of the Swiss Space Office at Bern, Switzerland, and the STI-Space Center at EPFL, Switzerland. The authors wish also to acknowledge CESI, Italy, for the support on solar panels design. 


\section{References}

[1] VAcCARO S., tORRES P., MOSIG J.R., ET Al.: 'Integrated solar panel antennas', Electron. Lett., 2000, 36, (5), pp. 390-391

[2] vaccaro S., torres P., mosig J.R., et al.: 'Combination of antennas and solar cells for satellite communications', Microw. Opt. Technol. Lett., MOTL, 2001, 29, (1), pp. $11-16$

[3] VACCARO S., MOSIG J.R., DE MAAGT P.: 'Application of the solar antenna 'SOLANT' concept to satellite platform'. Proc. 25th ESA Antenna Workshop on Satellite Antenna Technology, Noordwijk, 18-20 September 2002, pp. 111-118

[4] VACCARO S., MOSIG J.R., DE MAAGT P.: 'Two advanced solar antenna 'SOLANT' designs for satellite and terrestrial communications", IEEE Trans. Antennas Propag., 2003, AP-51, (8), pp. 2028-2034
[5] VAcCaro S., MOSig J.R., DE MAAGT P.: 'Making planar antennas out of solar cells', Electron. Lett., 2002, 38, (17), pp. $945-947$

[6] Vaccaro S., Mosig J.R., DE MAagt P.: 'Patch and slot antennas integrating high efficiency GaAs solar cells for space applications'. Journées Internationales de Nice sur les antennes (JINA'2002), Nice, 12-14 Novembre 2002, vol. 2, pp. 117-120

[7] http://www.ohb-system.de/gb/Transport/cosmos. html

[8] EVANS G.: 'Antenna measurement techniques' (Artech House, Norwood, MA, 1990)

[9] HUANG J.: 'A technique for an array to generate circular polarization with linearly polarized elements', IEEE Trans. Antennas Propag., 1986, AP-34, pp. 1113-1124 